\title{
Immediate Oral Rehabilitation of Atrophic Mandible
}

Case Report

Batista Mendes GC $^{1 *}$, Padovan $\mathrm{LEM}^{2}$, da Silva WS ${ }^{1}$, Ribeiro-Junior PD 3

${ }^{1}$ Postgraduate Student, PhD Program in Oral and Maxillofacial Surgery, Sagrado Coração University Bauru, São Paulo, Brazil.

${ }^{2}$ Professor, Department of Post-Graduation, Latin American Institute of Dental Research and Education (ILAPEO), Curitiba, Paraná, Brazil.

${ }^{3}$ Professor, Department of Oral and Maxillofacial Surgery, Sagrado Coração University Bauru, São Paulo, Brazil.

\section{Abstract}

The oral rehabilitation using osseointegrated implants in atrophic mandibles (AM) with severe bone resorption remains a surgical and prosthetic challenge, due to the risk of mandible fracture during implant surgery or under functional loading, paresthesia and pain. A patient with severe atrophic mandible was treated using a 2.0 locking system reconstruction plate combined with dental implants, in immediate loading system. Surgery was performed through intraoral approach and preserving mental nerve bundle integrity. After 36 months of clinical and radiographic postoperative control, it was found that this alternative treatment was safe, effective and had minimal morbidity. This technique enabled the patient to quickly recover masticatory function, rehabilitating the patient in a predictable manner.

Keywords: Dental Implants; Atrophic Mandible; Oral Rehabilitation; Bone Plates; Immediate Loading.

\section{Introduction}

Patients with mandibular atrophy usually present problems regarding the use of removable complete dentures, especially lack of stability. The usual complaints are difficult to eat and speak, lose the support of adjacent soft tissue, changes in facial appearance and pain caused by prosthetic trauma and by imbalance of the stomatognathic system [1].

The total rehabilitation of edentulous mandibles with osseointegrated implants (OI) was the first kind of treatment in the studies carried out by Professor P-I Branemark, with great success, predictability and efficiency. However, when dealing with severely atrophied mandibles, this treatment is less predictable, and there is an increased risk of intercurrences [2].

The functional rehabilitation of mandibles with severe bone resorption remains a surgical and prosthetic challenge due to the extreme decrease in supporting bone structure and the progressive nature of the process of resorption [3].

The aim of this study was to present a technique for immediate oral rehabilitation using OI and internal rigid fixation, in a patient with Luhr's [4] class III atrophic mandible.

\section{Case Report}

A 68-year-old man, was referred to a private clinic with a complaint of instability of the lower removable total denture and symptoms of pain in the jaw when chewing. Intraoral examination revealed severe resorption of the upper and lower alveolar ridge, and the presence of two transitional intra-osseous implants in the region between the mental foramina. Periimplantitis was diagnosed and one of the implants was severely mobile.

A cone beam tomography (Figure 1a, b, c, d) showed a severe atrophic mandible, with less than $5 \mathrm{~mm}$ thick in the posterior region, less than $7 \mathrm{~mm}$ height in the inter-foramina region.

The proposed treatment was the removal of the existing intraosseous implants, the placement of a $2.0-\mathrm{mm}$ locking system reconstruction plate extending from the right to the left side of the jaw, and a simultaneous placement of OIs in the region between the mental foramina, followed by the installation of a full-arch

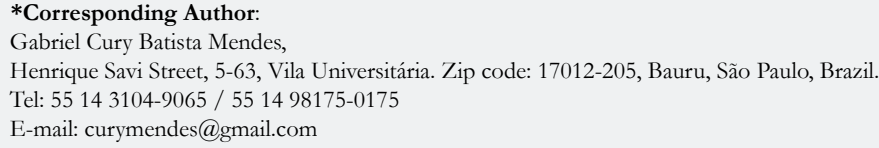

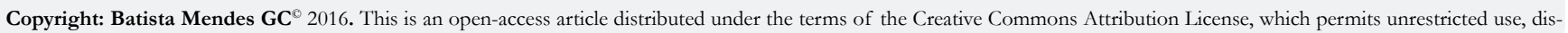
tribution and reproduction in any medium, provided the original author and source are credited. 
Figure 1. Pre operative CT scans. (a, b, c, d) CT scans showing severe mandibular resorption, main in posterior region. Note the exposure of the mental foramen at the same level as the mandibular bone crest level, the reason why the patient's mastication was painful due to the use of mucosa-supported denture.

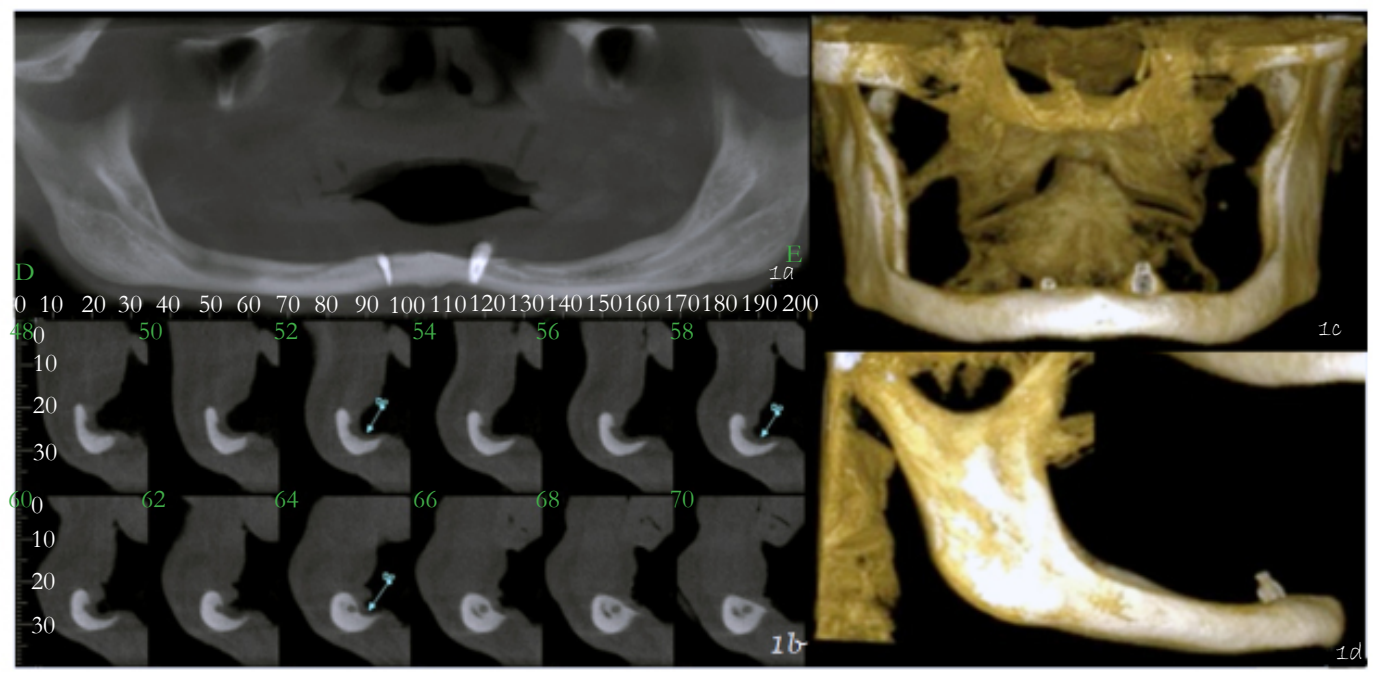

fixed hybrid prosthesis in immediate loading system.

Reverse prosthetic planning was carried out, and a multifunctional surgical guide was manufactured. On the biomodel, a previous adjustment of the reconstruction plate was carried out, and an acrylic osseous guide was made for the placement of the implants and the reconstruction plate (Figure 2a).

Surgical procedure was performed under general anesthesia. The access used was an intra-oral incision on the bone crest of the mandible extending from the right to the left mandibular angle, with preservation of the neurovascular bundle bilaterally. Bilateral submandibular trans-cutaneous access with approximately 1 centimetre long were made for the placement of a $2.0 \mathrm{~mm}$ locking reconstruction plate (Synthes, Swiss) and eight $2.0 \mathrm{~mm}$ screws, five of them in the body and angle areas below to alveolar canal bilateraly and three of them between the mental canals, for fixation of the reconstruction plate (Figure $2 \mathrm{~b}$ ).

After the plate was placed in correct position, perforations were made for the installation of dental implants in the region between mental foramina and between the $2.0 \mathrm{~mm}$ screws previously placed in this area. A depth probe and $2.0 \mathrm{~mm}$ burs were used to check if after a $7 \mathrm{~mm}$ depth drill the cortical bone of the mandibular base had been reached. Thus, four surgical OI sites were created for the installation of $3.5 \times 7 \mathrm{~mm}$ cylindrical dental implants with Cone Morse prosthetic connection (Neodent System, Curitiba, Brazil). As the initial stability of the OI was greater than 45 for all of the implants, prosthetic abutments were placed.

After discharge from the anesthesiology, transferral impressions were made using the multifunctional guide for the sequence of prosthetic procedures. Two days after surgery, a hybrid dentalgingival (acrilic and metal) fixed prosthesis was installed in the lower jaw and a denture in the upper jaw (Figure 2c).

The postoperative follow-up visits took place weekly during the first month and then monthly for a year. Currently, the control is carried out every six months totalling 36 months of post-treatment (Figure 2d). There was no pain in function, no paraesthesia and the vertical dimension was restored.

\section{Discussion}

Many alternatives for the treatment of atrophic mandible are reported in the literature, such as transmandibular implantation, endosteal dental implants, the use of short implants, osteogenic distraction, and grafting procedures [1].

Of all the above-mentioned techniques, the use of short implants is the one with less morbidity [5] and was indicated for the case described in this report. However, there is a risk of a mandibular fracture with this kind of treatment alone $[6,7]$, since the implant placement can weaken the already-compromised atrophic mandible [7]. As a means of strengthening the jaw, a 2.0-mm locking system reconstruction plate was used. To reduce surgical time and the morbidity of the procedure, the plate was previously adapted to a biomodel, allowing surgery to be performed mainly through intra-oral access rather than large extraoral incisions.

Using the same biomodel, the placement of the osseointegrated implants and plate's screws were also previously established, so that the screws in the anterior region did not interfere with the placement of the implants or with the inferior alveolar nerve bundle. Thus, an acrylic osseous guide was made to determine the position of both the OIs and the plate.

This alternative treatment of severely atrophic mandible proves to be effective, with less morbidity than other procedures described, and may be indicated in cases of severe mandibular resorption with the risk of a mandibular fracture after or during implant placement. This treatment proved to be safe and effective, allowing the patient to quickly return to full masticatory function.

\section{Acknowledgements}

Authors would like to thank the support of Neodent Implant System, Curitiba, PR, Brazil. 
Figure 2. Treatment sequence. (a) Reconstruction plate adapted to biomodel, and surgical osseous guide to aid in the placement of the implants and the plate during the surgery. (b) Intraoral photograph during the surgery, showing adapted and screwed plate, four implants placed in the inter-foramina region, abutments and it's protection cylinders installed. Note the integrity of the mental nerve bundle. (c) Prosthesis placed 48 hours after the surgery: an upper complete denture and a lower fixed hybrid prostheses, 3 months after. (d) 36 months follow-up panoramic radiograph.

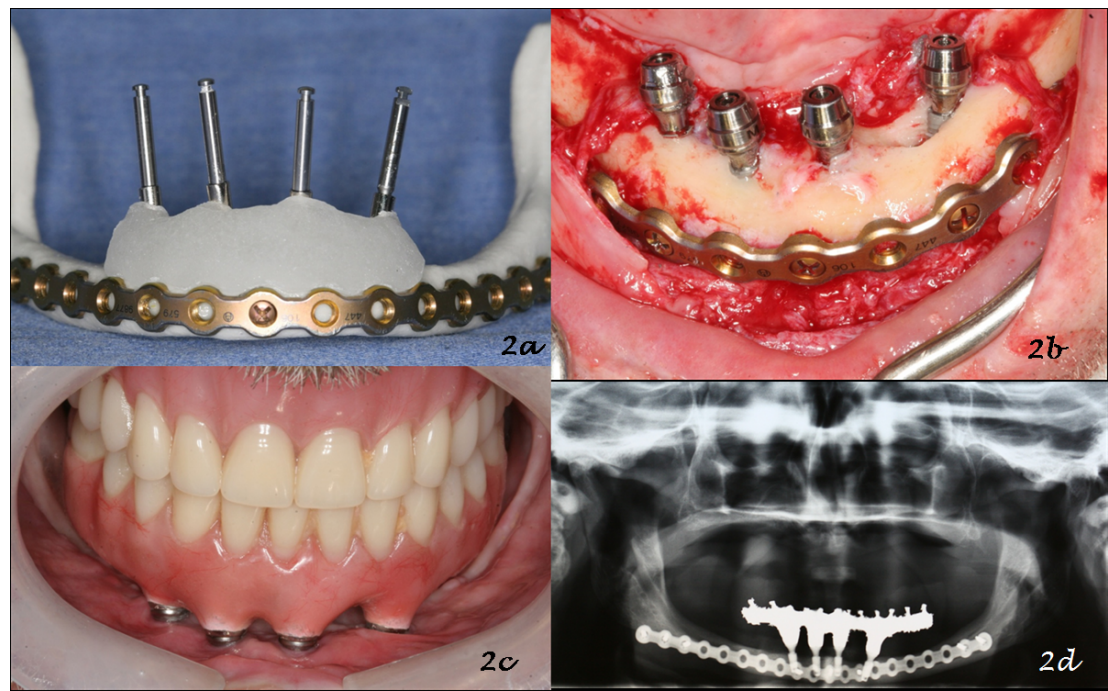

\section{References}

[1]. Stellingsma C, Vissink A, Meijer HJA, Kuiper C, Raghoebar GM (2004) Implantology and the Severely Resorbed Edentulous Mandible. Crit Rev Oral Biol Med 15(4): 240-248.

[2]. Raghoebar GM, Stellingsma K, Batenburg RH, Vissink A (2000) Etiology and management of mandibular fractures associated with endosteal implants in the atrophic mandible. Oral Surg Oral Med Oral Pathol Oral Radiol Endod 89(5): 553-559

[3]. Bell RB, Blakey GH, White RP, Hillebrand DG, Molina A (2002) Staged reconstruction of the severely atrophic mandible with autogenous bone graft and endosteal implants. J Oral Maxillofac Surg 60(10): 1135-1141.

[4]. Luhr HG, Reidick T, Merten HA (1996) Results of treatment of fractures of the atrophic edentulous mandible by compression plating: a retrospective evaluation of 84 consecutive cases. J Oral Maxillofac Surg 54(3): 250-254.

[5]. Keller EE (1995) Reconstruction of the severely atrophic edentulous mandible with endosseous implants: a 10-year longitudinal study. J Oral Maxillofac Surg 53(3): 305-320.

[6]. Mason ME, Triplett RG, Van Sickels JE, Parel SM (1990) Mandibular fractures through endosseous cylinder implants: report of cases and review. J Oral Maxillofac Surg 48(3): 311-317.

[7]. Chrcanovic BR, Custódio AL (2009) Mandibular fractures associated with endosteal implants. Oral Maxillofac Surg 13(4): 231-238. 